\title{
Costos de no calidad asociados a infección de sitio operatorio en poscesareadas, Colombia, 2020.
}

\section{Costs of doing things incorrectly associated with infections at the operating room post cesarean in Colombia 2020}

\author{
Solmaira Lora $\mathrm{H}^{1}$, Mónica Ocampo $\mathrm{R}^{2}$, Enalbis Espitia $\mathrm{C}^{3}$ (이 \\ RESUMEN
}

Objetivo: Determinar los costos de no calidad en una institución de salud de Colombia, en pacientes postquirúrgicas de cesárea que presentaron Infección en Sitio Operatorio (ISO). Materiales y métodos: Estudio descriptivo transversal, que estima los costos de no calidad de cuatro pacientes postquirúrgicas de cesárea que reingresaron a la institución por presentar infección en sitio quirúrgico. Los datos fueron proporcionados por el departamento de Calidad y Seguridad del Paciente, con base en los eventos adversos reportados en el período comprendido en el primer semestre del año 2020. Los costos hospitalarios, fueron clasificados de la siguiente manera: asistenciales, administrativas y de otro tipo, se calcularon desde que la paciente reingresó hasta que fue dada de alta. Resultados: El 100\% de las mujeres intervenidas tienen edades que oscilan entre los 25 y 30 años, con nivel socioeconómico de estrato 1 (89.5\%); promedio de 5 días de estancia hospitalaria al reingreso; los costos del reingreso hospitalario son mayores con un promedio de $\$ 304.943$ (IC, 149.672 - \$460.215); la realización de la cesárea muestra un costo promedio de $\$ 184.918$. Con relación a los costos asistenciales, el mayor promedio de la realización de la cesárea se encuentra en las consultas con \$527.224, seguido de los gastos quirúrgicos con un promedio de $\$ 415.395$ y de recurso humano con $\$ 298.236$; mientras que para el reingreso, el mayor costo se presentó en recursos humanos con $\$ 1.133 .298$ en promedio, seguido de las consultas con $\$ 645.681$ y los costos de habitación con $\$ 439.613$. Conclusión: Los costos de no calidad afecta el presupuesto de la institución, colocando en riesgo la estabilidad financiera y la imagen corporativa. Es importante realizar seguimiento a la adherencia de los protocolos, guías de atención, y paquetes instruccionales de buenas prácticas.

Palabras clave: Costos de la Atención en Salud, calidad, eventos adversos, seguridad del paciente.

\begin{abstract}
Objective: To determine the non-quality costs in a health institution in Colombia, in post-surgical cesarean section patients who presented infection at the surgical site (SSI). Materials and methods: Cross-sectional descriptive study, which estimates the non-quality costs of four postoperative cesarean section patients who were readmitted to the institution due to infection at the surgical site. The data were provided by the Department of Quality and Patient Safety, based on the adverse events reported in the period comprised in the first semester of 2020. Hospital costs were classified as follows: care, administrative and other type, were calculated from the time the patient was readmitted until she was discharged. Results: $100 \%$ of the intervened women have ages ranging between 25 and 30 years, with a socioeconomic level of stratum $1(89.5 \%)$; average of 5 days of hospital stay upon readmission; the costs of hospital readmission are higher with an average of \$304,943 (Cl, 149,672 - \$460,215); performing a cesarean section shows an average cost of $\$ 184,918$. In relation to healthcare costs, the highest average for cesarean section is found in consultations with $\$ 527,224$, followed by surgical expenses with an average of $\$ 415,395$ and human resources with \$298,236; while for re-entry, the highest cost was in human resources with $\$ 1,133,298$ on average, followed by consultations with $\$ 645,681$ and room costs with $\$ 439,613$. Conclusion: Non-quality costs affect the institution's budget, putting financial stability and corporate image at risk. It is important to monitor adherence to protocols, care guides, and instructional packages of good practices.
\end{abstract}

Keywords: Health Care Costs, quality, adverse events, patient safety

Citación (Vancouver)

Costos de no calidad asociados a infección de sitio operatorio en poscesareadas, Colombia, 2020. Rev Avances en Salud; 2021. (5)1:11-19 e. doi: 10.21897/25394622.2518

(c) 2020. Universidad de Cordoba. Este es un artículo de acceso abierto distribuido bajo los términos de la licencia Creative Commons Attribution License, que permite el uso ilimitado, distribución y reproducción en cualquier medio, siempre que el autor original y la fuente se acreditan.

'Especialista en Auditoría Corporativa de la Calidad. Docente. Universidad de Córdoba.

${ }^{2}$ Magister en Salud Pública, Enfermera, Docente Programa Administración en Salud,.

${ }^{3}$ Magister en Administración de Organizaciones, Enfermera, Docente Programa Administración en Salud, Universidad de Córdoba.

Carrera. 6 \#76-103 Montería-Córdoba, e-mail: enalbisespitia@correo.unicordoba.edu.co 


\section{INTRODUCCIÓN}

Las infecciones asociadas a la atención en salud (IAAS), se adquieren durante la estancia hospitalaria; clínicamente el paciente no presenta ningún signo o síntoma, y tampoco se encuentra en período de incubación al momento del ingreso para una atención en salud $(1,2)$; son frecuentes en los países en vía de desarrollo, y representan una carga epidemiológica en los países de altos ingresos (3). La infección de la herida quirúrgica, es una causa común de las IAAS $(4,5,6)$ y afectan la piel, tejidos u órganos de la zona donde se llevó a cabo la intervención quirúrgica (7); los pacientes que desarrollan esta infección, tienen una alta probabilidad de reingreso hospitalario (2), y en muchos de ellos, su vida se encuentra en peligro (8). Debido a que las infecciones de la herida quirúrgica, afectan la economía familiar, y aumentan los costos de no calidad en las instituciones de salud, al prolongar las estancias hospitalarias, e incluso en algunas ocasiones provocar discapacidad a largo plazo $(4,6)$ y resistencia a los antibióticos $(8)$, se requiere la implementación de estrategias por su elevada prevalencia, gravedad, incremento de costos directos e indirectos en salud y disponibilidad de medidas preventivas científicamente probadas (7). Con respecto a la cesárea, es considerada como una de las cirugía más realizada en los hospitales de segundo y tercer nivel de atención (9), y la infección en sitio quirúrgico, es una de las principales complicaciones que pueden presentarse; (10) lo que genera un aumento de la estancia hospitalaria y mayores gastos en la atención en salud (11), algunos autores afirman que la incidencia varía de un país a otro (12, 13), y al respecto, la Organización Mundial de Salud (OMS), manifiesta que en África, hasta un $20 \%$ de las mujeres sometidas a cesárea sufren infecciones de la herida quirúrgica (8), otros estudios han encontrado que la infección de la pared abdominal ocurre entre el $3 \%$ y $16 \%$ de las operaciones cesárea (14).
Por otra parte, los costos de no calidad en salud, hace referencia a los que incurre las instituciones debido a las ineficiencias o incumplimientos evitables (15) durante la atención en salud, siendo estos significativamente altos; de ahí la importancia de su medición y análisis, con el fin de apoyar la toma de decisiones para el mejoramiento y control, con la finalidad de garantizar la viabilidad financiera y la obtención de excedentes operativos para el desarrollo científico y tecnológico de la institución (16). Por lo anterior, el equipo investigador consideró importante realizar un estudio para determinar los costos de no calidad en una institución de salud de Colombia, en pacientes postquirúrgicas de cesárea que presentaron infección en sitio operatorio.

\section{MATERIALES Y MÉTODOS}

Se realizó un estudio descriptivo transversal, en una institución de salud de tercer nivel de complejidad de Colombia, durante el periodo comprendido de enero a junio del año 2020. La población y muestra estuvo conformada por cuatro pacientes postquirúrgicas de cesárea que reingresaron a la institución por presentar infección de la herida quirúrgica, y fueron reportadas al Sistema de Vigilancia en Salud Pública del Ministerio de Salud y Protección Social de Colombia (17).

Se estudiaron los costos en pesos colombianos, cuando se realizó la intervención quirúrgica y se compararon con los generados durante el reingreso hospitalario, clasificándolos en tres grupos (administrativos, asistenciales y otros). Los costos asistenciales hacen referencia a los medicamentos administrados, ocupación de la habitación, insumos médico quirúrgicos utilizados, personal involucrado en la atención (horas-médico; horas-enfermería; consulta por nutricionista, entre otros), gastos quirúrgicos, procedimientos diagnósticos realizados y valor de la consulta por especialista.

En cuanto a los costos administrativos, son los causados por los servicios públicos (agua y 
energía) y alimentación, requeridos para ofrecer una atención de calidad. Los otros costos hacen referencia a los que incurre el paciente o familiares por el acompañamiento que deben hacerle mientras se encuentra hospitalizado, entre los que se encuentran: pasajes, estadía, comida, entre otros.

La información fue proporcionada por el área de Calidad y Seguridad del Paciente, posteriormente se elaboró una hoja en Microsoft Excel para calcular los costos generados durante la atención de cada paciente. Se realizaron cálculos estadísticos descriptivos de centralidad y dispersión, así como el cálculo del intervalo de confianza para el promedio por medio de t-student a un nivel de confianza al 95\%. Los resultados se muestran en tablas.

\section{RESULTADOS}

Del total de casos analizados se observó, que el 75\% de las puérperas participantes en el estudio se encuentran entre los 24 y 30 años; con procedencia igual de la zona urbana y rural (50\%); nivel educativo en su mayoría bachilleres (50\%), técnicos y profesionales 25\% cada uno; en cuanto al nivel socioeconómico, el $50 \%$ son de estrato I, mientras que el estrato II y III, aportan cada uno el 25\%; la estancia hospitalaria promedio en el reingreso fue de 5 días. (Tabla 1)

\section{Tabla 1. Aspectos sociodemográficos}

\begin{tabular}{|c|c|c|}
\hline Característica & $\mathbf{n}$ & $\%$ \\
\hline \multicolumn{3}{|l|}{ Edad (años) } \\
\hline$<17$ & 0 & $0 \%$ \\
\hline $18-23$ & 0 & $0 \%$ \\
\hline $24-30$ & 3 & $75 \%$ \\
\hline $31-36$ & 1 & $25 \%$ \\
\hline$>36$ & 0 & $0 \%$ \\
\hline \multicolumn{3}{|l|}{ Procedencia } \\
\hline Urbana & 2 & $50 \%$ \\
\hline Rural & 2 & $50 \%$ \\
\hline \multicolumn{3}{|l|}{ Nivel educativo } \\
\hline Primaria & 0 & $0 \%$ \\
\hline Secundaria & 2 & $50 \%$ \\
\hline Técnico & 1 & $25 \%$ \\
\hline Profesional & 1 & $25 \%$ \\
\hline \multicolumn{3}{|l|}{ Nivel socioeconómico } \\
\hline Estrato I & 3 & $75 \%$ \\
\hline Estrato II & 1 & $25 \%$ \\
\hline Estrato III & 0 & $0 \%$ \\
\hline
\end{tabular}

Fuente: Elaboración propia 
En cuanto a los costos económicos generados, se evidenció que los relacionados con el reingreso hospitalario se duplicó con respecto a las atenciones seguras; si comparamos las 4 atenciones el total fue de $\$ 7.396 .716$, y los reingresos suman un valor de $\$ 15.857 .060$ pesos colombianos. (Tabla 2).

Tabla 2. Costos por pacientes de cesáreas realizadas y reingreso hospitalario

\begin{tabular}{ll|ll|ll|l|} 
Paciente & \multicolumn{3}{c}{ Asistenciales } & \multicolumn{2}{c}{ Administrativos } & \multicolumn{2}{l}{ Otro tipo } \\
& $\begin{array}{l}\text { Realización } \\
\text { de } \\
\text { la cesárea } \\
(\$)\end{array}$ & $\begin{array}{l}\text { Reingreso } \\
\text { hospitalario } \\
(\$)\end{array}$ & $\begin{array}{l}\text { Realización } \\
\text { de la cesárea } \\
(\$)\end{array}$ & $\begin{array}{l}\text { Reingreso } \\
\text { hospitalario } \\
(\$)\end{array}$ & $\begin{array}{l}\text { Realización } \\
\text { de la cesárea } \\
(\$)\end{array}$ & $\begin{array}{l}\text { Reingreso } \\
\text { hospitalario } \\
(\$)\end{array}$ \\
\hline 1 & 1.444 .376 & 2.441 .309 & 708.000 & 3.696 .000 & 0 & 0 \\
\hline 2 & 1.847 .619 & 3.103 .834 & 57.435 & 208.307 & 0 & 946.000 \\
\hline 3 & 1.234 .805 & 2.625 .985 & 27.238 & 176.274 & 0 & 75.000 \\
\hline TOTAL & 2.016 .775 & 2.126 .242 & 60.468 & 308.109 & 0 & 150.000 \\
\hline
\end{tabular}

Fuente: Elaboración propia

El costo promedio asistencial de los cuatro pacientes fue de $\$ 300.731$ pesos colombianos, con desviación estándar de \$505.123, e intervalo de confianza de $\$ 165.458$ - $\$ 436.004$, donde se espera se encuentren los costos de otros pacientes seleccionados al azar. (Tabla 3) Para los costos administrativos se encontró una media de $\$ 218.410$ pesos colombianos, desviación de $\$ 501.763$, intervalo de confianza con límite inferior de $\$ 13.151$ y límite superior de $\$ 423.668$, y para otro tipo de costos se
Tabla 3. Estadísticos del tipo de costo presentó un promedio de $\$ 48.792$ pesos colombianos, intervalo de confianza de $\$ 0$ a $\$ 119.989$. Cabe resaltar que en los diferentes tipos de costos se observó una variabilidad alta. (Tabla 3)

Por otra parte, el coeficiente de variación explica que tanto varían los datos; y como este es superior al $100 \%$, se entiende que la variabilidad de los costos en estos cuatro pacientes es grande. (Tabla 3)

\begin{tabular}{lc|cc|cc|} 
Tipo de costo & $\begin{array}{c}\text { Media } \\
(\$)\end{array}$ & $\begin{array}{c}\text { Desv. } \\
\text { Estándar } \\
(\$)\end{array}$ & $\begin{array}{c}\text { Error } \\
\text { Desviación } \\
(\$)\end{array}$ & $\begin{array}{c}\text { Intervalo de } \\
\text { Confianza }(\$)\end{array}$ & $\begin{array}{c}\text { Coef. } \\
\text { Variación } \\
(\$)\end{array}$ \\
\hline Asistenciales & 300.731 & 505.123 & 67.500 & $165.458-436.004$ & $168 \%$ \\
\hline Administrativos & 218.410 & 501.763 & 102.422 & $13.151-423.668$ & $230 \%$ \\
\hline Otro tipo & 48.792 & 174.045 & 35.527 & $0-119.989$ & $357 \%$ \\
\hline
\end{tabular}

Fuente: Elaboración propia 
En la tabla 4 se observa el comportamiento de los tipos de costos de acuerdo con el concepto de cada uno, al respecto se encontró que los costos más elevados se presentaron por reingreso hospitalario en cada tipo de costo. En cuanto a los costos asistenciales, se tiene que por realización de cesárea el promedio fue de \$233.699 (IC, \$79.577 - \$387.821) con una significancia del 5\%; mientras que para el reingreso hospitalario en el mismo tipo de costo el promedio es de $\$ 367.763$ (IC, \$137.533 - \$597.993). (Tabla 4)

Para el caso de los costos administrativos se evidencia una mayor diferencia con $\$ 71.095$ (IC, $\$ 0$ - \$163.588) para la realización de la cesárea y un promedio de \$365.724 (IC, \$0 - \$795.659). Para el otro tipo de costos, no se reportaron en las realizaciones de cesárea y para el reingreso se encontró un promedio de \$97.583 (IC, \$0 - \$250.789). (Tabla 4)

El termino (IC) hace referencia a intervalo de confianza.

\begin{tabular}{|lc|cc|cc|c|}
\hline \multicolumn{1}{c}{ Concepto } & \multicolumn{2}{c}{ Asistenciales } & \multicolumn{2}{c}{ Administrativos } & \multicolumn{2}{c|}{ Otro tipo } \\
\hline Realización de la cesárea & 233.699 & \pm 154.122 & 71.095 & \pm 92.493 & 0 & \pm 0 \\
\hline Reingreso hospitalario & 367.763 & \pm 230.230 & 365.724 & \pm 429.935 & 97.583 & \pm 153.206 \\
\hline
\end{tabular}

Fuente: Elaboración propia

La tabla 5 muestra las estadísticas de los costos por concepto y se resalta que en general el reingreso hospitalario tiene mayores costos con un promedio de \$304.943 (IC, 149.672 \$460.215); la realización de la cesárea tiene un costo promedio de \$184.918 (IC, \$73.658 - \$296.178); en otras palabras, los costos de reingreso hospitalario tienen una mayor desviación media que realizar una cesárea.

\section{Tabla 5. Estadísticos por concepto}

\begin{tabular}{lc|cc|cc}
\multicolumn{1}{c|}{ Concepto } & Media & $\begin{array}{c}\text { Desv. } \\
\text { Estándar }\end{array}$ & $\begin{array}{c}\text { Error } \\
\text { Desviación }\end{array}$ & $\begin{array}{c}\text { Intervalo de } \\
\text { Confianza }\end{array}$ & $\begin{array}{c}\text { Coef. } \\
\text { Variación }\end{array}$ \\
$\begin{array}{l}\text { Realización } \\
\text { de la cesárea }\end{array}$ & 184.918 & 347.887 & 55.005 & $73.658-296.178$ & $188 \%$ \\
$\begin{array}{l}\text { Reingreso } \\
\text { hospitalario }\end{array}$ & 304.943 & 557.724 & 77.342 & $149.672-460.215$ & $183 \%$
\end{tabular}

Fuente: Elaboración propia

Analizandolos costos de forma desagregada, se observó que en los asistenciales, el mayor promedio de la realización de la cesárea se encuentra en las consultas con \$527.224 (IC, \$0 - \$2.107.143), seguido de los gastos quirúrgicos con un promedio de $\$ 415.395$ (IC, $\$ 0$ - \$906.055) y recurso humano con \$298.236 (IC, \$0 - \$661.671); mientras que para el reingreso, el mayor costo se presentó en recursos humanos con \$1.133.298 en promedio (IC, \$0 $\$ 2.425 .223)$, seguido de las consultas con $\$ 645.681$ (IC, $\$ 0-\$ 2.221 .556$ ) y los costos de habitación con $\$ 439.613$ (IC, $\$ 0$ - \$1.056.596). 
En los administrativos, el mayor promedio se encontró en la energía, con un costo promedio de (\$148.471) (IC, \$0 - \$521.564) para la realización de la cesárea, y para el reingreso hospitalario un promedio de \$594.138 (IC, \$0 - \$2.086.076). Mientras que el menor costo para ambos conceptos fue la alimentación, con un promedio de \$10.000 (IC, \$1.784 - \$18.216) para cesárea y \$84.000 (IC, \$49.143 - \$118.857) para reingreso.

Para el caso de otro tipo de gastos, se encontró que para la realización de cesárea no se incurrieron en costos, mientras que para reingreso se incurrieron en paciente $y$ familiares, con costos medios de $\$ 211.500$ $(I C, \$ 0-\$ 884.493)$ y $\$ 81.250$ (IC, \$0 $\$ 180.688$ ) respectivamente. (Tabla 6).

Tabla 6. Concepto y tipo de costo

\begin{tabular}{|c|c|c|c|}
\hline \multirow[b]{2}{*}{ Costos } & \multirow[b]{2}{*}{ Concepto } & \multicolumn{2}{|c|}{ Costos } \\
\hline & & $\begin{array}{l}\text { Realización de la } \\
\text { cesárea }\end{array}$ & $\begin{array}{c}\text { Reingreso } \\
\text { hospitalario }\end{array}$ \\
\hline \multirow{7}{*}{ 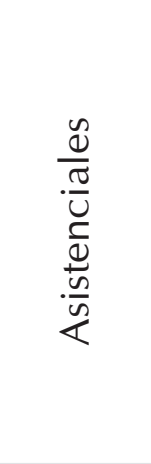 } & Medicamentos & $86.918 \pm 99.439$ & $208.144 \pm 272.953$ \\
\hline & Habitación & $123.343 \pm 154.739$ & $439.613 \pm 616.983$ \\
\hline & Insumos médicos & $62.209 \pm 72.627$ & $39.840 \pm 61.915$ \\
\hline & Recurso Humano & $298.236 \pm 363.435$ & $1.133 .298 \pm 1.291 .925$ \\
\hline & Gastos quirúrgicos & $415.395 \pm 490.660$ & $0 \pm 0$ \\
\hline & P. Diagnósticos & $122.570 \pm 139.119$ & $107.768 \pm 169.937$ \\
\hline & $\begin{array}{l}\text { Valor de consultas } \\
\text { por especialista }\end{array}$ & $527.224 \pm 1.579 .919$ & $645.681 \pm 1.575 .876$ \\
\hline \multirow{3}{*}{ 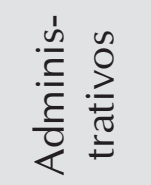 } & Agua & $54.814 \pm 154.024$ & $419.035 \pm 1.252 .640$ \\
\hline & Energía & $148.471 \pm 373.093$ & $594.138 \pm 1.491 .938$ \\
\hline & Alimentación & $10.000 \pm 8.216$ & $84.000 \pm 34.857$ \\
\hline \multirow{2}{*}{$\stackrel{ }{0} \stackrel{\circ}{=}$} & Paciente & $0 \pm 0$ & $81.250 \pm 99.437$ \\
\hline & Familiares & $0 \pm 0$ & $211.500 \pm 672.993$ \\
\hline
\end{tabular}

Fuente: Elaboración propia 


\section{DISCUSIÓN}

Dentro de los eventos adversos prevenibles que con mayor frecuencia se presentan, son las IAAS; y la infección en el sitio quirúrgico hace parte de estas, siendo una de las principales complicaciones en pacientes obstétricas $(10,18)$; considerado como un importante problema de salud e indicador de la calidad de la atención en salud (10).

Por otra parte, los costos injustificados que se producen por fallas en la atención a los pacientes, son conocidos como costos de no calidad, y su medición y análisis es de gran importancia en las organizaciones de salud (16), debido a que proporcionan información del ahorro que se podría conseguir invirtiendo en programas de prevención y control en las IAAS (19). En este estudio se encontró que el costo por reingreso hospitalario fue mucho mayor con relación al generado durante la realización de la cesárea, resultados que coinciden con hallazgos previos realizados por otros autores a nivel nacional (19) e internacional (20).

Al analizar las variables sociodemográficas, con respecto a la distribución por grupo de edad, se observó que las puérperas sujetos de estudio, se encuentran en su mayoría entre los 24 y 30 años, con resultados similares reportados por otros autores (11), lo cual puede deberse, a que esta es la edad de mayor fertilidad (11). En lo relacionado a la posición social, pertenecen a un nivel económico bajo; similares hallazgos fueron encontrados por Aguiar et al (21); y en cuanto al nivel académico, el grado de instrucción más frecuente fue la secundaria, resultados que coinciden con un estudio realizado en Lima-Perú (22).

Teniendo en cuenta el orden de los costos producidos, se encontró que en el reingreso hospitalario, el mayor costo se presentó en recursos humanos, seguido del valor de las consultas por especialista y en tercer lugar los generados por la hospitalización. Con respecto a la duración de la internación, se ha encontrado que las infecciones del sitio quirúrgico prolongan la estancia hospitalaria (21); por tanto, también los costos de la atención y posibilidades de complicaciones en el paciente (23); resultados que también fueron hallados en este estudio, con un aumento de 5 días-camas por ISQ en las poscesareadas, generando un costo adicional.

De acuerdo a la clasificación de los costos, en este estudio se encontró que los más elevados se presentaron por reingreso hospitalario en cada uno de ellos. En los costos asistenciales, la realización de cesárea, el promedio fue de \$233.699 (IC, \$79.577 - \$387.821); mientras que durante el reingreso hospitalario en el mismo tipo de costo el promedio fue de $\$ 367.763$ (IC, \$137.533 - \$597.993). Con relación a los administrativos, se evidenció una mayor diferencia con \$71.095 (IC, \$0 - \$163.588) para la realización de la cesárea y un promedio de $\$ 365.724$ (IC, \$0 - \$795.659) durante el reingreso, y finalmente para el otro tipo de costos, no se reportaron en las realizaciones de cesárea y para el reingreso se encontró un promedio de \$97.583 (IC, \$0 - \$250.789); estos resultados pueden orientar a los administradores de las instituciones de salud, acerca de los costos de no calidad que se pueden generar cuando no se ofrece una atención segura, y la importancia de fomentar una cultura de seguridad del paciente en los colaboradores de la institución (20).

\section{CONCLUSIONES}

Luego de analizar los 4 eventos, se encontró, que los costos de no calidad afecta la institución, colocando en riesgo la estabilidad financiera y la imagen corporativa; sin olvidar el daño que se hace al usuario y su familia, entre el que se encuentra, la separación del recién nacido de su madre, cuando esta se encuentra hospitalizada. Por tanto, es importante realizar seguimiento a la adherencia de los protocolos, guías de atención, y paquetes instruccionales de buenas prácticas. Además se debe monitorizar, la curación de la herida quirúrgica en casa, el saneamiento ambiental, administración del tratamiento, y alimentación. 


\section{REFERENCIAS}

1. Rodríguez, G. Santana, S. Villar del Campo, M. Martín, R. Martínez, J. Gil, A. Evaluación de la adecuación de la profilaxis antibiótica en cirugía ortopédica y traumatológica. Elsevier. (2015). 28(1): 17-20. https://doi. org/10.1016/j.eimc.2008.11.011

2. Colás-Ruiz, E., Del-Moral-Luque, J. A., Gil-Yonte, P., Fernández-Cebrián, J. M., Alonso-García, M., Villar-del-Campo, M. C., ... \& Rodríguez-Caravaca, G. Incidencia de infección de sitio quirúrgico y factores de riesgo en cirugía de recto. Estudio de cohortes prospectivo. Cirugía Española, 2018; 96(10), 640-647

3. Febré Naldy, Díaz-Toro Felipe, Cea-Nettig Ximena. Nuevas recomendaciones de la OMS sobre medidas para la prevención de infección de sitio operatorio. Rev. chil. infectol. [Internet]. 2017 Oct [citado 2021 Jun 01] ; 34( 5 ): 523-524. Disponible en: http://www.scielo.cl/scielo.php?script=sci_arttext\&pid=S0716-10182017000500523\&In$g=e s$

4. Díaz, C. Las infecciones nosocomiales, un problema vigente. Rev. Cuerpo méd. HNAAA. (2016). 9(1): 4-5

5. Barrachina, R. L., de la Cruz Tabares, E., \& Collado, I. D. L. T. G. (2021). Incidencia de infección de localización quirúrgica en cirugía de colon según metodología RENAVE: estudio prospectivo 2017-2019. Cirugía Española, 99(1), 34-40

6. Nájera, G. F. R., Barquero, F. A. C., \& Bermúdez, C. A. U. (2020). Factores de riesgo y prevención de infecciones del sitio quirúrgico. Revista Médica Sinergia, 5(04), 1-10. Colás-Ruiz, E., Del-Moral-Luque, J. A., Gil-Yonte, P., Fernández-Cebrián, J. M., Alonso-García, M., Villar-del-Campo, M. C., ... \& Rodríguez-Caravaca, G. (2018). Incidencia de infección de sitio quirúrgico y factores de riesgo en cirugía de recto. Estudio de cohortes prospectivo. Cirugía Española, 96(10), 640-647

7. Gómez-Romero, F. J., Fernández-Prada, M., \& Navarro-Gracia, J. F. (2017). Prevención de la infección de sitio quirúrgico: análisis y revisión narrativa de las guías de práctica clínica. Cirugía Española, 95(9), 490502

8. Organización Mundial de la Salud. La OMS recomienda 29 formas de detener las infecciones quirúrgicas y evitar microorganismos multirresistentes. 2016. Disponible en: https://www.who.int/es/news/ item/03-11-2016-who-recommends-29-waysto-stop-surgical-infections-and-avoid-superbugs\#: : :text=Las $\% 20$ infecciones $\% 20$ quir $\%$ C3\%BArgicas $\% 2 \mathrm{C} \% 20$ causadas $\% 20$ por,los $\% 20$ pacientes $\% 20$ operados $\% 20$ sufren\%20infecciones

9. Méndez, D. N., \& Núñez, D. C. (2017). Análisis de la cesárea como fuente de costo. Medisan, 21(08), 1008-1017\}

10. Ramírez Salinas Yamilia, Zayas Illas Arnaldo, Infante del Rey Solmary, Ramírez Salinas Yanilia M, Mesa Castellanos Iveet, Montoto Mayor Vicente. Surgical site infection in puerperal women with caesarean. Rev Cubana Obstet Ginecol [Internet]. 2016 Mar [citado 2021 Jun 02] ; 42( 1 ). Disponible en: http://scielo.sld.cu/scielo.php?script=sci_ arttext\&pid=S0138-600X2016000100005\&In$\mathrm{g}=\mathrm{es}$

11. Frias Chang Norla Virgen, Begué Dalmau Nuris de las Mercedes, Martí Rodríguez Luis Armando, Leyva Frias Norla, Méndez Leyva Leonor. Infección del sitio quirúrgico poscesárea. MEDISAN [Internet]. 2016 Mayo [citado 2021 Jun 02] ; 20 ( 5 ): 596-603. Disponible en: http://scielo.sld.cu/scielo.php?script=sci_ arttext\&pid=S1029-30192016000500002\&In$\mathrm{g}=\mathrm{es}$

12. Calderón León MF, Calle Morillo LV, Jaña Lozada JR, Parodi R, Hidalgo L. Infección puerperal poscesárea vs infección puerperal posparto en el hospital ginecoobstétrico Enrique C. Sotomayor. Rev Med FCM-UCSG. 2011;17(4):238-43.

13. Organización Panamericana de la Salud. Vigilancia epidemiológica de las infecciones asociadas a la atención de la salud en el puerperio. [Documento en Internet]. 2014. Disponible en: http://www.paho.org/hq/index. php?option=com_docman\&task=doc_view\&gid=25547\& \&temid 
15. Barrios Junco, Susana. "Costos de Calidad y Costos de no Calidad: una decisión de mercado." 2013.

16. Arango Cardona, Luis Javier. "Importancia de los costos de la calidad y no calidad en las empresas de salud como herramienta de gestión para la competitividad." Revista Ean 67 (2009): 75-94.

17. Ministerio de Salud y Protección Social. Sistema de Vigilancia en Salud Pública. Disponible en: https://www.minsalud.gov.co/ salud/Paginas/SIVIGILA.aspx\#: :text=EI\%20 Sistema\%20de\%20Salud $\% 20 \mathrm{P} \%$ C3\%BAblica,pr\%C3\%A1 ctica $\% 20$ de $\% 201$ a $\% 20$ salud $\% 20 p \%$ C3\%BAblica

18. Rommy, D. Q. Factores de riesgo asociados a infección del sitio operatorio post cesárea del Hospital Nacional Edgardo Rebagliati-2017. Revista Médica Panacea. 2019; 8(1).

19. Restrepo, L. A. R., Suárez, J. C. C., \& Martínez, M. E. G. (2011). Costo de las infecciones del sitio operatorio en una institución de alta complejidad. Medellín Colombia, 2008-2009. Revista CES Salud Pública, 2(2), 169-189.

20. Gutiérrez-Mendoza L, Torres-Montes A, Soria-Orozco M, Padrón-Salas A, Ramírez-Hernández $\mathrm{M}$. Costos de eventos adversos graves en un Hospital Comunitario de Enseñanza en México. 2015; 83(3):211-216. DOI: 10.1016/j.circir.2015.05.013

21. Aguiar da Cruz Lidiane, Vieira Freitas Lydia, Moura Barbosa Régia Christina, De Souza Gomes Linicarla Fabiole, Teixeira Vasconcelos Camila Moreira. Infección de herida operatoria tras cesárea en un hospital público de Fortaleza. Enferm. glob. [Internet]. 2013 Ene [citado 2021 Jun 15] ; 12( 29 ): 105-117. Disponible en: http://scielo.isciii.es/scielo.php?script=sci_arttext\&pi$\mathrm{d}=\mathrm{S} 1695-61412013000100005 \&$ Ing=es.

22. Richter Soto, L. (2016). Factores de riesgo asociaciones a infección de herida operatoria post cesárea en el Hospital Vitarte periodo 2013 a Junio 2015.Disponible en: http://168.121.49.87/bitstream/handle/ urp/746/Richter_L.pdf?sequence=1\&isAllowed=y
23. Peralta, M. H. V., León, I. V. V., López, K. J. M., Santacruz, B. A. U., \& Benítez, S. N. G. (2018). ¿ Qué nos dicen los estudios de incidencia de infección de la herida quirúrgica?. Enfermería Investiga: Investigación, Vinculación, Docencia y Gestión, 3(1), 15-22 